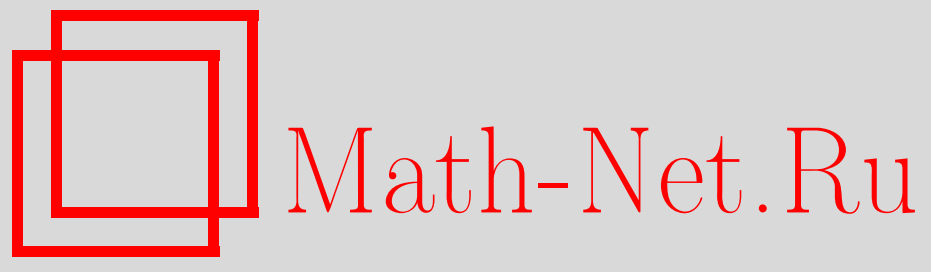

А. С. Горский, Судаковский скейлинг и дуальность между калибровочными полями и струнами, ТМФ, 2007, том 150, номер 2, 204-218

DOI: https://doi.org/10.4213/tmf5974

Использование Общероссийского математического портала Math-Net.Ru подразумевает, что вы прочитали и согласны с пользовательским соглашением http://www . mathnet.ru/rus/agreement

Параметры загрузки:

IP: 44.207 .124 .84

26 апреля 2023 г., 06:44:33

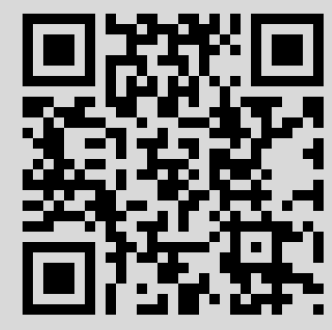




\title{
СУДАКОВСКИЙ СКЕЙЛИНГ И ДУАЛЬНОСТЬ МЕЖДУ КАЛИБРОВОЧНЫМИ ПОЛЯМИ И СТРУНАМИ
}

\begin{abstract}
Исследуются аномальные размерности (супер)конформных операторов Вильсона в режимах слабой и сильной связи с использованием симметрий интегрируемых систем с обеих сторон соответствия калибровочные поля/струны. Изучается происхождение однологарифмического асимптотического поведения длинных операторов/струн в пределе большого лоренцевского спина.
\end{abstract}

Ключевые слова: спиновые цепочки, калибровочные теории.

\section{1. ВВЕДЕНИЕ}

Аномальные размерности операторов теории Янга-Миллса с большим лоренцевым спином играют важную роль в разнообразных явлениях в калибровочных теориях, использующих судаковский формфактор. Последние исследования дуальности между калибровочными полями и струнами [1] подают надежду на возможное точное вычисление всего ряда теории возмущений. В настоящей статье, основанной на работе [2], исследуются свойства аномальных размерностей операторов типа

$$
\mathcal{O}_{N, L}(0)=\sum_{n_{1}+\cdots+n_{L}=N} c_{n_{1} \ldots n_{L}} \operatorname{tr}\left\{D_{+}^{n_{1}} X(0) D_{+}^{n_{2}} X(0) \ldots D_{+}^{n_{L}} X(0)\right\}
$$

с обеих сторон соответствия калибровочные поля/струны в пределе большого твиста $L$ и лоренцева спина $N$. В этом выражении через $X(0)$ обозначена так называемая "регулярная" компонента квантовых полей определенной спиральности в соответствующей калибровочной теории, $D_{+}=D_{\mu} n^{\mu}$ представляет собой проекцию ковариантной производной на световой конус, $n_{\mu}^{2}=0$. Коэффициенты разложения $c_{n_{1} \ldots n_{L}}$ фиксируются с помощью условия, утверждающего, что $\mathcal{O}_{N, L}(0)$ обладает собственным масштабным поведением, т.е. выражение (1.1) должно быть собственным состоянием однопетлевого оператора дилатации. Со стороны теории струн соответствующие односледовые операторы Вильсона дуальны сложенной струне, вращающейся с большим угловым моментом $N$ в подпространстве $A d S_{3}$ пространства анти-де Ситтера $(\mathrm{AdS})$ и бустированной вдоль некоторого большого круга на

* Институт теоретической и экспериментальной физики, Москва, Россия. E-mail: gorsky@itep.ru 
сфере большого углового момента $L$ [3]. Энергия $E$ такой классической струнной конфигурации определяет ведущий асимптотический член разложения аномальной размерности $\gamma(\lambda)=E-L-N$ дуального оператора Вильсона в $\mathcal{N}=4$ теории супер-Янга-Миллса (СЯМ) в режиме сильной связи в планарном приближении. Из струнной теории следуют четкие предсказания о виде $\gamma(\lambda)$ как функции от $L$ и $N$. Можно получить, что $\gamma(\lambda)$ ведет себя по-разному в следующих трех режимах [4], [3]:

- при $N \ll L$ в пределе "коротких" струн

$$
\gamma(\lambda)=\lambda \frac{m^{2}}{2} \frac{N}{L^{2}}+\cdots
$$

- при $N \gg L$ в пределе “длинных" струн

$$
\gamma(\lambda)= \begin{cases}\frac{\lambda}{2 \pi^{2}} \frac{m^{2}}{L} \ln ^{2} \frac{N}{L}+\cdots & \text { для } \xi_{\mathrm{str}}<1, \\ \frac{\sqrt{\lambda}}{\pi} m \ln \frac{N}{\sqrt{\lambda}}+\cdots & \text { для } \xi_{\mathrm{str}} \gg 1,\end{cases}
$$

где параметр $\xi_{\mathrm{str}}=\lambda \ln ^{2}(N / L) / L^{2}$. Целое число $m$ здесь задает число раз, которое струна оказывается сложенной сама на себя. Минимальная аномальная размерность отвечает однократно сложенной струне, $m=1$. В этом случае при $\xi_{\text {str }} \gg 1$ ведущий асимптотический член для $\gamma(\lambda)$ не зависит от твиста $L$ и оказывается одним и тем же для всех операторов с $L=2$. При $\xi_{\mathrm{str}}<1$ роль твиста $L$ состоит в создании “окна БМН", т.е. области в пространстве параметров, в которой аномальная размерность допускает разложение в ряд по степеням константы связи БеренштейнаМалдачены-Настасе (БМН) $\lambda^{\prime} \equiv \lambda /(\pi L)^{2}$ [5]. Считается, что несколько первых членов в разложении $\gamma(\lambda)$ по степеням $\lambda^{\prime}$ должны совпадать с соответствующими выражениями для аномальных размерностей операторов Вильсона с твистом $L$ и спином $N$, полученных в $\mathcal{N}=4$ теории СЯМ в режиме слабой связи [6].

С точки зрения калибровочной теории вычисление аномальных размерностей для операторов старших твистов с большим лоренцевским спином представляет собой исключительно сложную задачу в общей теории Янга-Миллса даже на уровне однопетлевых вычислений ввиду большого размера матрицы смешивания. Эту проблему удается, однако, преодолеть, учитывая скрытую симметрию интегрируемости оператора дилатаций [7], которая отображает матрицу однопетлевого смешивания для операторов Вильсона с твистом $L$, принадлежащих так называемому голоморфному $S L(2)$-сектору, в гамильтониан магнетика Гейзенберга длины $L$ и спина $s$, задаваемого конформным спином квантовых полей (см. обзор [8]). Это наблюдение позволяет вычислить точный спектр собственных значений аномальных размерностей операторов Вильсона произвольного твиста и лоренцевского спина в интегрируемых секторах теории Янга-Миллса. В $\mathcal{N}=4$ теории СЯМ можно отождествить на уровне однопетлевой точности минимальную аномальную размерность операторов Вильсона, построенных из $L$ скалярных полей и несущих лоренцевский спин $N$, с минимальной энергией спектра собственных значений $S L(2)$-магнетика Гейзенберга длины $L$ с полным спином $N+L s, s=1 / 2$ [9]. Соответствие калибровочные поля/струны предполагает, что минимальная аномальная размерность, определенная таким образом, должна удовлетворять соотношениям (1.2), (1.3) в термодинамическом пределе $L \rightarrow \infty$. 
Из формул (1.2), (1.3) следует, что аномальные размерности операторов старших твистов зависят от "скрытого" параметра $\xi_{\mathrm{str}}=\lambda \ln ^{2}(N / L) / L^{2}$, а их поведение в режиме сильной связи оказывается различным при $\xi_{\mathrm{str}}<1$ и при $\xi_{\mathrm{str}} \gg 1$. При $\xi_{\text {str }} \gg 1$ аномальная размерность не допускает пертурбативного разложения по константе БМН $\lambda^{\prime}$ и имеет масштабное поведение $\sim \ln N$. В результате исследований анзаца Бете для спиновой цепочки $S L(2)$ в термодинамическом пределе $L \gg 1$ с точки зрения калибровочных теорий в работе [9] было получено выражение для $\gamma(\lambda)$, которое совпадало с $(1.2)$ в пределе коротких струн и с первым соотношением в формуле (1.3) в пределе длинных струн. Авторы, тем не менее, не обнаружили ни какого-либо проявления второго (логарифмического) режима поведения, ни появления нового параметра, сходного с $\xi_{\mathrm{str}}$. Это наблюдение противоречит нашим предположениям о том, что минимальная аномальная размерность операторов старших твистов должна иметь масштабное логарифмическое поведение во всех петлевых поправках при $N \rightarrow \infty$. Цель настоящего исследования состоит в получении логарифмического масштабного поведения аномальных размерностей как в калибровочной, так и в струнной теории и в осознании физического смысла параметра $\xi_{\text {str }}$ и его калибровочного аналога $\xi$.

Мы перепроверим вычисления энергии $S L(2)$-магнетика Гейзенберга со спином $s=1 / 2,1,3 / 2$, используя в качестве основного приема метод $Q$-оператора Бакстера [10], и покажем, что однопетлевая аномальная размерность в интегрируемых секторах теории Янга-Миллса обладает следующим масштабным поведением в термодинамическом пределе $L \rightarrow \infty$ :

- при $N \ll L$

$$
\gamma(\lambda)=\lambda \frac{m^{2}}{4 s} \frac{N}{L^{2}}+\cdots
$$

- при $N \gg L$

$$
\gamma(\lambda)= \begin{cases}\frac{\lambda}{2 \pi^{2}} \frac{m^{2}}{L} \ln ^{2} \frac{N}{L}+\cdots & \text { для } \xi<1, \\ \frac{\lambda}{2 \pi^{2}} m \ln N+\cdots & \text { для } \xi \gg 1,\end{cases}
$$

в зависимости от параметра $\xi=\ln (N / L) / L$. Здесь $s$ совпадает с конформным спином поля, входящего в оператор Вильсона, т.е. $s=1 / 2,1,3 / 2$ соответственно для скалярного поля, поля фермионного партнера и поля глюона. Для скалярных операторов уравнение (1.4) и первое из соотношений (1.5) совпадают с соответствующими выражениями в (1.2), (1.3). Заметим, что аномальная размерность в (1.5) не зависит от спина $s$ при $N \gg L$, что предполагает универсальность двух режимов в формуле (1.5) для всех калибровочных теорий. Это действительно так при $\xi \gg 1$ ввиду того, что коэффициент при $2 \ln N$ совпадает с аномальной размерностью излома в режиме слабой связи.

При $N \gg L$ и $\xi<1$ однопетлевая аномальная размерность проявляет новое, дважды логарифмическое поведение (1.5). Впервые этот результат был получен из соображений теории струн [3], а в дальнейшем был воспроизведен вычислениями с точки зрения калибровочных теорий [9]. Возникает естественный вопрос: можно ли получить похожие выражения, если обратиться к старшим петлям, и можно ли их просуммировать во всех петлях? Соответствие между калибровочными полями 
и струнами предполагает, что в $\mathcal{N}=4$ теории СЯМ аномальная размерность в области значений $N \gg L \gg 1$ допускает разложение типа БМН (с $\lambda^{\prime}=\lambda /(\pi L)^{2}$ и $\xi=\ln (N / L) / L)$

$$
\gamma(\lambda)=L \sum_{n=1}^{\infty}\left(\lambda^{\prime} \ln ^{2} \frac{N}{L}\right)^{n} c_{n}(\xi)+\cdots,
$$

в котором коэффициентные функции $c_{n}(\xi)$ не зависят от константы связи и при $\xi \rightarrow 0$ и $\xi \rightarrow \infty$ имеют асимптотики

$$
c_{n}(\xi)=c_{0, n}+c_{1, n} \xi+O\left(\xi^{2}\right), \quad c_{n}(\xi)=O\left(\frac{1}{\xi^{2 n-1}}\right),
$$

$c_{0, n}=(-1)^{n}(-1 / 2)_{n} / n$ !. На уровне одной петли из уравнения (1.6) следуют (при $m=1$ ) оба соотношения (1.5). Кроме того, для $\xi \rightarrow \infty$ коэффициент при $\lambda^{n}$ в правой части разложения (1.6) имеет масштабное поведение вида $\sim \ln N$ и задает $n$-петлевую поправку к аномальной размерности конфигурации типа излома в режиме слабой связи. В режиме сильной связи после подстановки $\xi=\xi_{\mathrm{str}}^{1 / 2} / \sqrt{\lambda}$ оказывается возможным просуммировать пертурбативное разложение из (1.6) по всем петлевым поправкам, что приводит (при $\lambda \rightarrow \infty$ и фиксированном значении $\left.\xi_{\text {str }}=\lambda \ln ^{2}(N / L) / L^{2}\right)$ к выражению

$$
\gamma(\lambda)=L \sum_{n=1}^{\infty}\left(\lambda^{\prime} \ln ^{2} \frac{N}{L}\right)^{n} c_{n}(0)+\cdots=L\left[\sqrt{1+\lambda^{\prime} \ln ^{2} \frac{N}{L}}-1\right]+\cdots
$$

где многоточие обозначает поправки следующих порядков. При $N \rightarrow \infty$ из этого выражения можно также получить ведущее асимптотическое поведение аномальной размерности в последнем из режимов, указанных в (1.3), и, следовательно, выражение для аномальной размерности в режиме сильной связи.

\section{2. СПЕКТР ЭНЕРГИИ ДЛЯ СПИНОВОЙ ЦЕПОЧКИ}

Наиболее эффективный способ квантования системы состоит в использовании решения уравнения Бакстера, которое в данном случае имеет вид

$$
(u+i s)^{L} Q(u+i)+(u-i s)^{L} Q(u-i)=t_{L}(u) Q(u)
$$

и задает энергию и импульс посредством соотношений

$$
\varepsilon=i(\ln Q(i s))^{\prime}-i(\ln Q(-i s))^{\prime}, \quad e^{i \theta}=\frac{Q(i s)}{Q(-i s)} .
$$

Удобно ввести эйкональную фазу:

$$
Q\left(\frac{x}{\eta}\right)=\eta^{-N} e^{S(x) / \eta}, \quad S(x)=\eta \sum_{k=1}^{N} \ln \left(x-\eta \lambda_{k}\right),
$$

где

$$
\eta=\frac{1}{N+L s}, \quad \beta=s L \eta=\frac{s L}{N+L s} .
$$


В квазиклассическом приближении с помощью разложения

$$
S(x)=S_{0}(x)+\eta S_{1}(x)+\cdots, \quad \hat{q}_{k}=\hat{q}_{k}^{(0)}+\eta \hat{q}_{k}^{(1)}+\cdots,
$$

где $\eta$ играет роль постоянной Планка, энергия спинового магнетика или, эквивалентно, однопетлевая аномальная размерность оператора Вильсона параметризуется комплексной кривой $\Gamma_{L}$. Род кривой $g=L-2$ задается длиной спиновой цепочки, в то время как ее модули определяются квантованными значениями сохраняющихся зарядов $\hat{q}_{k}$, которые, в свою очередь, зависят от набора целых чисел $\ell_{1}, \ldots, \ell_{L-1}$. Выбирая различные значения этих чисел, можно рассматривать различные участки спектра.

В данной работе исследуются собственные состояния, на которых достигается минимально возможная энергия при фиксированном полном спине $N$. Для собственных состояний, отвечающих минимальной энергии, все отрезки, кроме двух, сжимаются в двойные точки, а корни Бете конденсируются на двух симметрично расположенных разрезах на вещественной оси, которые обозначаются $[-a,-b]$ и $[b, a]$. Тогда спектральная кривая принимает вид

$$
y^{2}=\tilde{y}^{2}\left[\frac{2}{x^{3}} \prod_{j}\left(1-\frac{x_{2 j}^{2}}{x^{2}}\right)\right]^{2}, \quad \tilde{y}^{2}=\left(x^{2}-a^{2}\right)\left(x^{2}-b^{2}\right),
$$

где было принято в расчет то, что точки ветвления возникают парами с противополжными знаками для состояний с минимальной энергией. Параметры $a, b$ и $x_{2 j}$ определяются из требования аналитичности функции $S_{0}^{\prime}(x)$ на комплексной $x$ плоскости с двумя симметрично расположенными разрезами на вещественной оси и с предписанным асимптотическим поведением функции при $x=0$ и $x \rightarrow \infty$ [11]. Это выражение находится в явном противоречии с тем хорошо известным обстоятельством, что аномальная размерность имеет логарифмическое масштабное поведение $\varepsilon \sim \ln N$ при $N \gg L$ с коэффициентом, не зависящим от $L$.

Рассмотрим в качестве примера собственные состояния с фиксированным значением $L, N \rightarrow \infty$, и старшими зарядами. В этом случае трансфер-матрица $\tau_{0}(x)$ представляет собой четный многочлен степени $L$, имеющий при $x \rightarrow 0$ поведение

$$
\tau_{0}(x) \sim \frac{\hat{q}_{L}^{(0)}}{x^{L}}
$$

если только $\hat{q}_{L}^{(0)} \neq 0$. При нулевом $\hat{q}_{L}^{(0)}$ асимптотическое поведение $\tau_{0}(x)$ задается первым неисчезающим зарядом $\hat{q}_{2 k}^{(0)} \neq 0$ с $2 k<L$. Можно найти главную асимптотику импульса при $x \rightarrow 0$ на верхнем листе:

$$
d p \sim i L \frac{d x}{x},
$$

что дает квазиимпульс $p(x) \sim i L \ln x$ и энергию

$$
\varepsilon \sim 2 L \ln \frac{L}{\beta} \sim 2 L \ln N
$$


Заметим, что коэффициент при $\ln N$ в правой части (2.9) задается главной асимптотикой трансфер-матрицы $(2.7)$ при $x \rightarrow 0$. Для $\hat{q}_{L}^{(0)} \neq 0$ этот коэффициент принимает свое максимально возможное значение. При $\hat{q}_{L}^{(0)}=\cdots=\hat{q}_{2 m+2}^{(0)}=0$ трансферматрица масштабируется как $\tau_{0}(x) \sim \hat{q}_{2 m}^{(0)} / x^{2 m}$ (напомним, что заряды с нечетными индексами обращаются в нуль), что дает

$$
\varepsilon \sim 4 m \ln N, \quad \hat{q}_{2 m+2}^{(0)}=\cdots=\hat{q}_{L}^{(0)}=0 .
$$

Значения остающихся зарядов $\hat{q}_{4}^{(0)}, \ldots, \hat{q}_{2 m}^{(0)}$ будут найдены ниже (см. уравнение (2.16)). На протяжении всей статьи нас будут интересовать только собственные состояния с минимальной энергией при заданном $N$. Они, очевидно, отвечают $m=1$, т.e.

$$
\varepsilon^{(m=1)} \sim 4 \ln N, \quad \hat{q}_{4}^{(0)}=\cdots=\hat{q}_{L}^{(0)}=0 .
$$

Здесь верхний индекс (0) относится к ведущему порядку квазиклассического разложения, и из $\hat{q}_{2 k}^{(0)}=0$ не обязательно следует, что $\hat{q}_{2 k}=0$, а только лишь то, что $\hat{q}_{2 k}=O(\eta)$ или, эквивалентно, $q_{2 k} \ll\left(-q_{2}\right)^{k}$.

Трансфер-матрица $\tau_{0}(x)$, отвечающая состоянию с минимальной энергией $(2.11)$, задается соотношением

$$
\tau_{0}^{(m=1)}(x)=2-\frac{1}{x^{2}},
$$

а спектральная кривая принимает вид

$$
\Gamma_{L}^{(m=1)}: \quad y^{2}=\left(x^{2}-\frac{1}{4}\right) \frac{4}{x^{4}} .
$$

Легко видеть, что $\Gamma_{L}^{(m=1)}$ совпадает с комплексной кривой спиновой цепочки длины 2, т.е. $\Gamma_{L=2}$. В самом деле, выбирая $\hat{q}_{2 m+1}^{(0)}=\cdots=\hat{q}_{L}^{(0)}=0$, можно эффективно редуцировать случай бесконечно длинной спиновой цепочки (при $L \rightarrow \infty$ ) к цепочке конечной длины $2 m$. Из выражения (2.13) можно заключить, что при $m=1$ все точки ветвления, кроме двух, концентрируются в начале отсчета, $b=x_{2 j}=0$, а две оставшиеся (разрешенные) точки ветвления совпадают с $\pm 1 / 2$. При $m \geqslant 2$ можно показать [12], что комплексная кривая, отвечающая соотношению (2.10), имеет вид

$$
\Gamma_{L}^{(m \geqslant 2)}: \quad y^{2}=\left(x^{2}-\frac{1}{4 m^{2}}\right) \frac{4}{x^{4}} \prod_{j=1}^{m-1}\left(1-\frac{x_{2 j}^{2}}{x^{2}}\right)^{2},
$$

$1 / x_{2 j}=2 m \cos (\pi j /(2 m))$. Сравнивая этот результат с кривой $(2.6)$, получим, что $a=1 /(2 m)$ и $b=0$, т.е. два разреза $[-a,-b]$ и $[b, a]$ сливаются в начале координат. Заметим, что $\Gamma_{L}^{(m \geqslant 2)}$ имеет ровно $2 m$ двойных точек, удовлетворяющих условиям $x_{2 j}^{2}>1 /(2 m)^{2}$, в то время как оставшиеся двойные точки, лежащие на отрезке $[-b, b]$ в выражении (2.6), конденсируются в точке $x=0$ при $b \rightarrow 0$.

Комплексные кривые $(2.13)$, (2.14) имеют нулевой род, и вследствие этого импульс $p^{\prime}(x)$ выражается в элементарных функциях. В самом деле, при подстановке

2 Теоретическая и математическая физика, т. 150, № 2, 2007 г. 
(2.13), (2.14) можно получить следующее выражение для импульса при произвльном $m$ :

$$
d p=-\frac{d x}{x \sqrt{x^{2}-1 /(2 m)^{2}}}, \quad p(x)=\int_{\infty}^{x} d p=2 m \arcsin \frac{1}{2 m x} .
$$

Отсюда для интегралов движения получаем

$$
\widehat{q}_{2 n}^{(0)}=\frac{2(-1)^{n} m^{1-2 n} \Gamma(m+n)}{\Gamma(2 n+1) \Gamma(m-n+1)} .
$$

Из выражения (2.15) можно заключить, что для $m=1$ и собственных состояний с минимальной энергией в пределе $N \rightarrow \infty$ и при фиксированном значении $L$ импульс $p^{\prime}(x)$ представляет собой аналитическую функцию в комплексной плоскости с корневым разрезом $[-1 / 2,1 / 2]$ и простым полюсом в начале отсчета. Это условие следует сравнить с аналитическими свойствами импульса в области фиксированых значений $N / L$, а в этом случае $p^{\prime}(x)$ имеет двойной полюс при $x=0$.

\section{3. КЛАССИЧЕСКИЕ СТРУНЫ В $A d S_{3} \times S^{1}$}

Обратимся теперь к анализу аномальных размерностей составных операторов с точки зрения теории струн. В соответствии с дуальностью между калибровочными полями и струнами операторы Вильсона отображаются в определенные струнные состояния, энергии которых отождествляются с масштабными размерностями операторов. Известно, что в $\mathcal{N}=4$ теории СЯМ операторы Вильсона с минимальными аномальными размерностями, обсуждаемые в предыдущих разделах, дуальны однократно сложенным струнам, вращающимся в секторе $A d S_{3} \times S^{1}$ струнной теории типа IIB [4], [3].

3.1. Сложенная вращающаяся струна. Для сложенной замкнутой струны, вращающейся в подпространствах $A d S_{3}$ и $S^{1}$, бозонная часть суперструнного действия имеет вид

$$
S=-\frac{\sqrt{\lambda}}{2 \pi} \int d^{2} \xi \sqrt{-\operatorname{det}\left\|G_{M N}(X) \partial_{a} X^{M} \partial_{b} X^{N}\right\|},
$$

где константа взаимодействия 'т Хоофта $\lambda=g^{2} N_{c}$ связана с произведением радиуса $R$ пространства AdS и натяжения струны $1 / \alpha^{\prime}: \sqrt{\lambda}=R^{2} / \alpha^{\prime}$, а метрика имеет вид

$$
d s^{2} \equiv G_{M N} d X^{M} d X^{N}=-\operatorname{ch}^{2} \rho d t^{2}+\operatorname{sh}^{2} \rho d \theta^{2}+d \rho^{2}+d \varphi^{2} .
$$

Здесь $t, \rho$ и $\theta$ - соответственно глобальное время, радиальная координата и угловая переменная в пространстве $A d S_{3}$, а $\varphi$ - угол в большом круге на сфере $S^{5}$. Координаты мирового листа струны $\xi^{a}=\left(\xi^{0}, \xi^{1}\right)$ выбраны таким образом, что

$$
t=\xi^{0}, \quad \theta=\omega \xi^{0}+\xi^{1}, \quad \varphi=\nu \xi^{0} .
$$

Здесь $\omega$ - угловая скорость пространства AdS, а $\nu$ - параметр буста центра масс сложенной струны на сфере $S^{5}$. Для жесткой сложенной струны радиальная переменная $\rho$ не зависит от $\xi^{0}$ и периодична по $\xi^{1}$, т.е. $\rho\left(\xi^{1}+2 \pi\right)=\rho\left(\xi^{1}\right)$. Зависимость 
$\rho=\rho\left(\xi^{1}\right)$ задается классическими струнными уравнениями движения [3], [4], [13]. Их решение описывает конфигурацию сложенной струны, составленной из четырех сегментов, протяженных в радиальном направлении от центра пространства AdS, $\rho=0$, в сторону его границы на расстояние $\rho_{0}$, которое зависит от угловых скоростей $(3.3)$ :

$$
\operatorname{coth}^{2} \rho_{0}=\frac{\omega^{2}-\nu^{2}}{1-\nu^{2}} \geqslant 1 .
$$

У струны имеются два излома в точке $\rho=\rho_{0}$; эти изломы отвечают за логарифмическое масштабирование аномальных размерностей операторов Вильсона в режиме сильной связи [13], [14].

В рамках соответствия калибровочные теории/струны аномальная размерность операторов (1.1) связана с энергией $E$ классической вращающейся струны:

$$
E=N+L+\gamma(\lambda)
$$

где лоренцев спин $N$ и твист $L$ заменяются соответствующими угловыми моментами пространств $A d S_{3}$ и $S^{1}$. Из действия Намбу-Гото (3.1) следует, что эти заряды равны

$$
E=\frac{2 \sqrt{\lambda}}{\pi} \frac{\sqrt{-\chi}}{\sqrt{1-\nu^{2}}} \mathbb{E}(\chi), \quad N=\frac{2 \sqrt{\lambda}}{\pi} \frac{\omega \sqrt{-\chi}}{\sqrt{1-\nu^{2}}}[\mathbb{E}(\chi)-\mathbb{K}(\chi)], \quad L=\frac{2 \sqrt{\lambda}}{\pi} \frac{\nu \sqrt{-\chi}}{\sqrt{1-\nu^{2}}} \mathbb{K}(\chi),
$$

где $\mathbb{K}(\chi)$ и $\mathbb{E}(\chi)$ - эллиптические функции соответственно первого и второго рода, а вспомогательный (имеющий отрицательное значение) параметр $\chi$ связан с расстоянием $\rho_{0}$ от центра пространства $\mathrm{AdS}$ до конечной точки струны, протяженной по радиальной координате:

$$
\chi=-\operatorname{sh}^{2} \rho_{0} .
$$

Исключая $\omega$ и $\nu$ и оставляя $\chi$, можно представить уравнения (3.6) в параметрическом виде [9]:

$$
\left(\frac{E}{\mathbb{E}(\chi)}\right)^{2}-\left(\frac{L}{\mathbb{K}(\chi)}\right)^{2}=-\frac{4 \lambda}{\pi^{2}} \chi, \quad\left(\frac{N}{\mathbb{E}(\chi)-\mathbb{K}(\chi)}\right)^{2}-\left(\frac{L}{\mathbb{K}(\chi)}\right)^{2}=\frac{4 \lambda}{\pi^{2}}(1-\chi) .
$$

С помощью этих соотношений можно анализировать поведение аномальной размерности $\gamma=E-N-L$ в режиме сильной связи в квазиклассическом пределе большого углового момента $L \rightarrow \infty, N \rightarrow \infty$, с фиксированными $N / \sqrt{\lambda}$ и $L / \sqrt{\lambda}$. Таким способом из уравнения (3.8) можно найти, что два предельных случая $N \ll L$ и $N \gg L$ отвечают соответственно случаям коротких и длинных струн, $\rho_{0} \ll 1$ и $\rho_{0} \gg 1$.

Хорошо известно [3], [4], что в пределе коротких струн $\rho_{0} \ll 1$ или, эквивалентно, $(-\chi) \ll 1$ аномальная размерность допускает БМН-разложение

$$
\gamma(\lambda)=L\left[\lambda^{\prime} \gamma^{(0)}+\left(\lambda^{\prime}\right)^{2} \gamma^{(1)}+\cdots\right]
$$

где $\gamma^{(0)}, \gamma^{(1)}, \ldots$ - функции отношения $N / L$ и $\lambda^{\prime}=\lambda /(\pi L)^{2}$ задают константу связи БМН. Подставляя $\chi=\chi_{0}+\lambda^{\prime} \chi_{1}+\cdots$ в уравнение (3.8) и приравнивая коэффициенты разложения в обеих частях этого уравнения по степеням $\lambda^{\prime}$, можно найти 
для $N / L \ll 1[3],[5],[9]$, что

$$
\gamma^{(0)}=\frac{\pi^{2}}{2} \frac{N}{L}+\cdots, \quad \gamma^{(1)}=-\frac{\pi^{4}}{8} \frac{N}{L}+\cdots .
$$

Член низшего порядка в разложении (3.9) совпадает с однопетлевым результатом с точки зрения калибровочной теории для $s=1 / 2$ и $m=1$ :

$$
\gamma^{(0)}=\frac{L}{8} \varepsilon
$$

Похожим образом в пределе длинных струн $\rho_{0} \rightarrow \infty$ или, эквивалентно, $(-\chi) \rightarrow \infty$, предполагая регулярность разложения $\chi$ по степеням $\lambda^{\prime}$, можно найти из уравнения (3.8), что параметр $\chi_{0}$ в ведущем порядке при $N / L \gg 1$ имеет поведение

$$
\chi_{0}=-\frac{N}{2 L} \ln \frac{N}{L} \text {. }
$$

Отсюда для коэффициентов разложения БМН в случае аномальной размерности (3.9) при $N / L \gg 1$ можно получить

$$
\begin{aligned}
& \gamma^{(0)}=\frac{1}{2} \ln ^{2}\left(-\chi_{0}\right)+\cdots=\frac{1}{2} \ln ^{2} \frac{N}{L}+\cdots, \\
& \gamma^{(1)}=-\frac{1}{8} \ln ^{4}\left(-\chi_{0}\right)+\cdots=-\frac{1}{8} \ln ^{4} \frac{N}{L}+\cdots,
\end{aligned}
$$

где многоточием обозначены члены следующих порядков при $\left(-\chi_{0}\right) \rightarrow \infty$. В соответствии с формулой (3.11) первое из соотношений (3.13) совпадает с однопетлевым выражением для аномальной размерности, полученным с помощью калибровочной теории. Более того, можно показать [9], что соотношение (3.11) (после преобразования Ландена, определенного ниже формулой (3.33)) выполняется при произвольных значениях $N / L$ с $\gamma^{(0)}$, заданным выражениями (3.8) и (3.9).

Вплоть до этого момента мы не наблюдаем ожидаемого поведения аномальной размерности $\gamma(\lambda) \sim \ln N$ при $N \gg L$. Напомним, что квазиклассическое разложение однопетлевой энергии $\varepsilon$ со стороны струнной теории было расходящимся при $N \gg L$, так что логарифмическое масштабирование $\varepsilon \sim \ln N$ может быть достигнуто путем пересуммирования полного ряда теории возмущений в квазиклассическом приближении по степеням параметра $\xi=\ln (N / L) / L$. Оказывается, что подобное явление имеет место в случае ряда (3.9) в струнной теории, хотя в этом случае параметр квазиклассического приближения оказывается другим - он равен

$$
\xi_{\text {str }}=\frac{\lambda}{L^{2}} \ln ^{2} \frac{N}{L}=\lambda \xi^{2}
$$

Это соответствие легко получить, сравнивая вклады в разложение $(3.9)$ от $\gamma^{(0)}$ и от $\gamma^{(1)}$ (см. (3.13)).

Чтобы просуммировать бесконечный ряд в выражении (3.9) для $\xi_{\mathrm{str}} \gg 1$, обратимся снова к (3.8) и перепишем его в виде

$$
\gamma(\lambda)=L\left[\sqrt{1-4 \lambda^{\prime} \chi \mathbb{K}^{2}(\chi)}-1+\Delta \gamma\right],
$$


где $\lambda^{\prime}=\lambda /(\pi L)^{2}$ и

$$
\Delta \gamma=-\frac{4 \lambda^{\prime} \mathbb{K}(\chi)[\mathbb{E}(\chi)-\mathbb{K}(\chi)]}{\sqrt{1-4 \lambda^{\prime} \chi \mathbb{K}^{2}(\chi)}+\sqrt{1-4 \lambda^{\prime}(\chi-1) \mathbb{K}^{2}(\chi)}} .
$$

В пределе длинных струн при $(-\chi) \rightarrow \infty$ выражение для $\gamma(\lambda)$ разлагается в ряд по степеням $4 \lambda^{\prime}(-\chi) \mathbb{K}^{2}(\chi) \sim \lambda^{\prime} \ln ^{2}(-\chi)$. Исследуя выражение для $\Delta \gamma$, найдем, что его вклад в $\gamma(\lambda)$ подавляется множителем $[\mathbb{E}(\chi)-\mathbb{K}(\chi)] /(-\chi \mathbb{K}(\chi)) \sim 1 / \ln (-\chi)$ по сравнению с первым членом в квадратных скобках в выражении (3.15). Отсюда следует, что в пределе $(-\chi) \rightarrow \infty$ при фиксированном $\lambda^{\prime} \ln ^{2}(-\chi)$ ведущее асимптотическое поведение $(3.15)$ имеет вид

$$
\gamma(\lambda)=L\left[\sqrt{1+\lambda^{\prime} \ln ^{2}(-\chi)}-1\right]+\cdots .
$$

Это выражение позволяет пересуммировать все дважды логарифмические поправки $\sim L\left[\lambda^{\prime} \ln ^{2}(-\chi)\right]^{n}$ к аномальной размерности $\gamma(\lambda)$ во всех порядках разложения по $n$ в режиме сильной связи. В частности, при $n=1,2$ получим (3.13). При $\lambda^{\prime} \ln ^{2}(-\chi) \ll 1$ можно разложить квадратный корень в выражении (3.17) и получить разложение типа БМН (3.9). В то же время при $\lambda^{\prime} \ln ^{2}(-\chi) \gg 1$ из соотношения (3.17) следует выражение

$$
\gamma(\lambda)=L \sqrt{\lambda^{\prime}} \ln (-\chi)+\cdots=\frac{\sqrt{\lambda}}{\pi} \ln (-\chi)+\cdots .
$$

При $(-\chi) \rightarrow \infty$ зависимость $\chi$ от частного $N / L$ и константы связи БМН $\lambda^{\prime}$ задается вторым из соотношений (3.8):

$$
\frac{1}{4 \chi^{2}}=\frac{L^{2}}{N^{2}}\left[\frac{1}{\ln ^{2}(-\chi)}+\lambda^{\prime}\right] .
$$

При $\xi_{\text {srt }}<1$ и $N \gg L$ из выражений (3.19) и (3.14) найдем, что

$$
\chi \sim \chi_{0}=-\frac{N}{2 L} \ln \frac{N}{L}
$$

и, таким образом,

$$
\gamma(\lambda)=L\left[\sqrt{1+\frac{\lambda}{(\pi L)^{2}} \ln ^{2}\left(\frac{N}{L}\right)}-1\right]+\cdots .
$$

При $\xi_{\text {srt }} \gg 1$ из соотношений (3.14) и (3.19) получаем $(-\chi) \sim N /\left(2 L \sqrt{\lambda^{\prime}}\right) \sim N / \sqrt{\lambda}$ и

$$
\gamma(\lambda)=\frac{\sqrt{\lambda}}{\pi} \ln \frac{N}{\sqrt{\lambda}}+\cdots
$$

Это выражение воспроизводит правильное асимптотическое поведение для аномальной размерности в режиме (1.3) и находится в согласии с результатами работ [3], [4], [13].

Коэффициент при $\ln N$ в выражении (3.21) задает ведущее асимптотическое поведение аномальной размерности конфигурации типа излома в режиме сильной связи 
в $\mathcal{N}=4$ теории СЯМ. Из соотношений (3.20), (3.21) следует замечательный результат: аномалия типа излома в режиме сильной связи может быть получена путем пересуммирования двойных логарифмических членов $\sim L\left[\lambda \ln ^{2}(N / L) / L^{2}\right]^{k}$ в разложении для аномальных размерностей операторов старших твистов $L$ и с большим лоренцевым спином $N \gg L$.

3.2. Двухразрезное решение в струнной сигма-модели. В предыдущем разделе было показано, что при $N \gg L$ зависимость аномальной размерности $\gamma(\lambda)$ от константы связи отличается в режимах $\xi_{\text {str }}<1$ и $\xi_{\text {str }} \gg 1$. В первом случае $\gamma(\lambda)$ допускает разложение типа БМН по степеням $\lambda / L^{2}$, в то время как во втором случае $\gamma(\lambda)$ зависит от $\lambda$ не аналитически и имеет логарифмическое поведение $\sim \sqrt{\lambda} \ln N$. Причина такой неаналитичности состоит в том, что при $\xi_{\text {str }} \gg 1$ концевые точки вращающейся струны приближаются к границе пространства $\mathrm{AdS}$, и ведущий вклад в энергию струны или, эквивалентно, в аномальную размерность (3.5) задается областью вблизи двух изломов, $\gamma(\lambda) \sim(\sqrt{\lambda} / \pi) 2 \rho_{0}$. Здесь радиальная координата излома имеет поведение $\rho_{0} \sim \ln (-\chi) / 2 \sim \ln (N / \sqrt{\lambda}) / 2$ при $\xi_{\text {str }} \gg 1$ (см. уравнения $(3.7),(3.19))$. Это явление весьма общее [13], [14] и имеет место для классических струнных конфигураций с произвольным числом изломов $n$. В этом случае каждый излом задает логарифмический вклад в энергию, и аномальная размерность имеет поведение $\gamma(\lambda)=n \sqrt{\lambda} /(2 \pi) \ln (N / \sqrt{\lambda})$ при $\xi_{\text {str }} \gg 1$. На первый взгляд, этот механизм представляется весьма отличным от сходного механизма в калибровочной теории. Напомним, что в калибровочной теории логарифмическое поведение аномальных размерностей в однопетлевом приближении при $N \gg L$ возникает при слиянии разрезов спектральной кривой для спиновой цепочки. Известно, что классические уравнения движения для струны в фоновом поле $A d S_{5} \times S^{5}$ точно интегрируемы, и их решения параметризуются спектральными кривыми. Более того, для струн, лежащих в части подпространства $A d S_{3} \times S^{1}$, спектральную кривую можно отождествить с комплексной гиперэллиптической кривой [15]. Для сложенной вращающейся замкнутой струны, обсуждаемой в предыдущем разделе, эта кривая задается эллиптической кривой с симметрично расположенными точками ветвления на оси вещественных чисел [16]:

$$
\Gamma_{\text {str }}: \quad y^{2}=\left(x^{2}-a_{\text {str }}^{2}\right)\left(x^{2}-b_{\text {str }}^{2}\right),
$$

где $a_{\text {str }}$ и $b_{\text {str }}$ принимают положительные значения $b_{\text {str }}<a_{\text {str }}$. В этом разделе мы преобразуем различные асимптотические режимы поведения аномальных размерностей при $N \gg L$, задаваемые выражениями (3.20) и (3.21), в свойства кривой (3.22) и вскроем механизм, ответственный за логарифмическое поведение (3.21).

Подобно классической спиновой цепочке $S L(2)$, классические струнные уравнения движения допускают представление Лакса, и их можно решить точно с помощью конструкции функции Бейкера-Ахиезера [15]. Как и прежде, множитель Блоха-Флоке для этой функции задает (квази)импульс $p(x)$, представляющий собой производящую функцию для сохраняющихся зарядов, включая энергию. Для 
сложенной вращающейся струнной конфигурации $p^{\prime}(x)$ представляет собой функцию, аналитическую в комплексной плоскости с двумя симметрично расположенными разрезами $\left[-a_{\text {str }},-b_{\text {str }}\right] \cup\left[b_{\text {str }}, a_{\text {str }}\right]$. Она однозначно задается требованием, утверждающим, что $d p=p^{\prime}(x) d x$ представляет собой мероморфный дифференциал на комплексной кривой (3.22), удовлетворяющий следующим условиям на верхнем листе римановой поверхности $\Gamma_{\text {str }}[16]$ :

- предписанному асимптотическому поведению на бесконечности и в начале отсчета:

$$
d p \stackrel{x \rightarrow \infty}{\sim}-2 \frac{d x}{x^{2}} \frac{E+N}{L}, \quad d p \stackrel{x \rightarrow 0}{\sim}-d x \frac{2}{\lambda^{\prime}} \frac{E-N}{L} l ;
$$

- условию однозначности:

$$
\int_{b_{\mathrm{str}}}^{a_{\mathrm{str}}} d p=0, \quad \int_{a_{\mathrm{str}}}^{\infty} d p=-\pi m
$$

- наличию двойных полюсов в точках $x= \pm \sqrt{\lambda} /(\pi L) \equiv \sqrt{\lambda^{\prime}}$ :

$$
d p \sim d x\left[-\frac{1}{\left(x \pm \sqrt{\lambda^{\prime}}\right)^{2}}+O\left(\left(x \pm \sqrt{\lambda^{\prime}}\right)^{0}\right)\right]
$$

Окончательное выражение для дифференциала $d p$ имеет вид

$$
d p=\frac{d x}{y}\left[\frac{y_{+}}{\left(x-\sqrt{\lambda^{\prime}}\right)^{2}}+\frac{y_{+}^{\prime}}{x-\sqrt{\lambda^{\prime}}}+\frac{y_{+}}{\left(x+\sqrt{\lambda^{\prime}}\right)^{2}}-\frac{y_{+}^{\prime}}{x+\sqrt{\lambda^{\prime}}}+C\right]
$$

где $y=y(x)$ задано соотношением $(3.22), y_{+}=y\left(\sqrt{\lambda^{\prime}}\right)$ и $y_{+}^{\prime}=y^{\prime}\left(\sqrt{\lambda^{\prime}}\right)$.

Уравнение (3.26) зависит от трех параметров: $a_{\mathrm{str}}, b_{\mathrm{str}}$ и $C$. Они фиксируются условиями нормировки (3.23) и (3.24):

$$
\begin{aligned}
b_{\mathrm{str}} & =\frac{1}{m \mathbb{K}(\tau)}\left[\left(1-\frac{\lambda^{\prime}}{a_{\mathrm{str}}^{2}}\right)\left(1-\frac{\lambda^{\prime}}{b_{\mathrm{str}}^{2}}\right)\right]^{-1 / 2}, \\
C & =-\frac{m a_{\mathrm{str}}}{2}\left[\mathbb{E}(\tau)-\frac{\lambda^{\prime}}{a_{\mathrm{str}}^{2}} \mathbb{K}(\tau)\right],
\end{aligned}
$$

где модулярный параметр $\tau=1-b_{\mathrm{str}}^{2} / a_{\mathrm{str}}^{2}$. Дополнительно из (3.23) можно найти следующие соотношения для $N / L$ и аномальной размерности $\gamma(\lambda)=E-N-L$ :

$$
\begin{aligned}
\frac{N}{L} & =\frac{m}{2}\left[\mathbb{E}(\tau)\left(a_{\text {str }}+\frac{\lambda^{\prime}}{b_{\text {str }}}\right)-\mathbb{K}(\tau)\left(b_{\text {str }}+\frac{\lambda^{\prime}}{a_{\text {str }}}\right)\right], \\
\frac{\gamma(\lambda)}{L} & =m\left[\mathbb{K}(\tau) b_{\text {str }}-\mathbb{E}(\tau) \frac{\lambda^{\prime}}{b_{\text {str }}}\right]-1,
\end{aligned}
$$

где $b_{\text {str }}$ определено первым из выражений $(3.27)$ и $a_{\text {str }}=b_{\text {str }} / \sqrt{1-\tau}$.

Исследуем зависимость аномальной размерности от констаны связи $\lambda^{\prime}=\lambda /(\pi L)^{2}$. Предполагая, что обе величины $b_{\text {str }}$ и $\tau$ допускают регулярное разложение по степеням $\lambda^{\prime}$, можно подставить эти разложения в (3.27), (3.28):

$$
b_{\mathrm{str}}=b_{\mathrm{str}}^{(0)}+\lambda^{\prime} b_{\mathrm{str}}^{(1)}+\cdots, \quad \tau=\tau^{(0)}+\lambda^{\prime} \tau^{(1)}+\cdots,
$$


и сравнить коэффициенты при различных степенях $\lambda^{\prime}$. Таким образом, в ведущем порядке получим

$$
b_{\mathrm{str}}^{(0)}=\frac{1}{m \mathbb{K}\left(\tau^{(0)}\right)}, \quad \frac{N}{L}=\frac{1}{2}\left[\frac{\mathbb{E}\left(\tau^{(0)}\right)}{\sqrt{1-\tau^{(0)}} \mathbb{K}\left(\tau^{(0)}\right)}-1\right],
$$

а все поправки к выражению (3.29) могут быть выражены через лидирующие члены. Тогда первые коэффициенты ряда БМН для аномальной размерности (3.9) задаются выражениями

$$
\begin{aligned}
& \gamma^{(0)}=\frac{m^{2}}{2} \mathbb{K}\left(\tau^{(0)}\right)\left[\left(2-\tau^{(0)}\right) \mathbb{K}\left(\tau^{(0)}\right)-2 \mathbb{E}\left(\tau^{(0)}\right)\right] \\
& \gamma^{(1)}=\frac{m^{4}}{8} \mathbb{K}^{3}\left(\tau^{(0)}\right)\left[\left(4\left(2-\tau^{(0)}\right) \sqrt{1-\tau^{(0)}}-\left(\tau^{(0)}\right)^{2}\right) \mathbb{K}\left(\tau^{(0)}\right)-8 \sqrt{1-\tau^{(0)}} \mathbb{E}\left(\tau^{(0)}\right)\right] .
\end{aligned}
$$

Вместе со вторым из соотношений (3.30) они задают параметрическую зависимость аномальной размерности (3.9) от $N / L$.

Функциональное выражение для аномальных размерностей (3.28) отличается от найденных в предыдущем пункте выражений (3.8). Соответствия можно достичь, если применить преобразование Ландена для модулярных параметров [9]

$$
\chi^{(0)}=-\frac{\left(1-\sqrt{1-\tau^{(0)}}\right)^{2}}{4 \sqrt{1-\tau^{(0)}}},
$$

после которого соотношения (3.8) и (3.28) совпадут в случае $m=1$. Напомним, что $(-\chi)$ зависит от радиальной координаты излома $\rho_{0}$, так что $\sqrt{1-\tau}=e^{-2 \rho_{0}}$, а предел длинных струн $\rho_{0} \rightarrow \infty$ отвечает пределу $\tau \rightarrow 1$. Предельный переход $\tau \rightarrow 1$ отвечает $a \rightarrow 1 /(2 m)$ и $b \rightarrow 0$ с точки зрения калибровочной теории. Вследствие этого два разреза $[-a,-b]$ и $[b, a]$ сливаются в начале отсчета, обеспечивая тем самым логарифмическое масштабное поведение для однопетлевой аномальной размерности (2.10). Изучим теперь предельный переход $\tau \rightarrow 1$ для полученных струнных выражений (3.27), (3.28).

Так как $\mathbb{K}(\tau) \sim-\ln (1-\tau) / 2$ при $\tau \rightarrow 1$, на основании первого равенства в $(3.27)$ следует ожидать, что $b_{\text {str }}$ в этом пределе обратится в нуль. Это действительно так при $\lambda^{\prime}=0$, в то время как при $\lambda^{\prime} \neq 0$ из (3.27) можно получить следующее: условие вещественности для $b_{\text {str }}$ предполагает, что его минимальное значение должно удовлетворять условию $b_{\mathrm{str}} \geqslant \sqrt{\lambda^{\prime}}$. Внимательно исследуя первое из соотношений $(3.27)$ при $\tau \rightarrow 1$, получим

$$
b_{\mathrm{str}}=\sqrt{\lambda^{\prime}+\frac{1}{m^{2} \ln ^{2} \sqrt{1-\tau}}} .
$$

При $\lambda^{\prime}=0$ это соотношение совпадает с однопетлевым выражением $b_{\mathrm{str}}^{(0)}=2 b / \beta$. Сравнивая (3.29) и (3.34), можно заключить, что поправки старших порядков в $b_{\mathrm{str}}$ отодвигают его минимальное значение от начала отсчета, предотвращая тем самым 
слияние двух разрезов $\left[-a_{\mathrm{str}},-b_{\mathrm{str}}\right]$ и $\left[b_{\mathrm{str}}, a_{\mathrm{str}}\right]$. Из (3.28) следует, что в пределе $\tau \rightarrow 1$

$$
\beta \approx \frac{L}{2 N}=\frac{\sqrt{1-\tau}}{m b_{\mathrm{str}}}+\cdots, \quad \gamma(\lambda)=L\left[-m b_{\mathrm{str}} \ln \sqrt{1-\tau}-1\right]+\cdots .
$$

Асимптотическое поведение этих выражений зависит от величины параметра $\xi_{\text {str }}=$ $\lambda^{\prime} \ln ^{2}(N / L)$ (см. уравнение (3.14)).

При $\xi_{\mathrm{str}}<1$ выражение для $b_{\mathrm{str}}$ (см. уравнение $(3.34)$ ) допускает разложение в ряд по степеням $\lambda^{\prime}$ и приводит с учетом условий (3.35) к выражениям

$$
b_{\text {str }}=\frac{1}{m \ln (N / L)}+\cdots, \quad a_{\text {str }}=\frac{N}{2 m L}+\cdots, \quad \sqrt{1-\tau}=\frac{L / N}{2 \ln (N / L)}+\cdots .
$$

Посредством подстановки (3.34) в (3.35) можно получить выражение для аномальной размерности $\gamma(\lambda)$, которое совпадает с (3.20) при $m=1$. Полезно сравнить положения разрезов $a$ и $b$ в калибровочной теории и $\hat{a}=\beta a_{\text {str }} / 2$ и $\hat{b}=\beta b_{\text {str }} / 2$ в струнной теории. Дополнительный множитель $\beta / 2$ возникает здесь ввиду различного определения локального параметра $x$ на спектральных кривых (2.6) и (3.22). Таким образом, можно найти, что $\hat{a}=1 /(2 m)+\cdots$ и $\hat{b}=(L / N) /(4 m \ln (N / L))+\cdots$, и эти формулы совпадают с аналогичными выражениями в калибровочной теории с точностью до однопетлевых поправок.

При $\xi_{\text {str }} \gg 1$ выражение для $b_{\text {str }}$ (см. (3.34)) оказывается неаналитическим по константе связи БМН $\lambda^{\prime}=\lambda /(\pi L)^{2}$ :

$$
b_{\text {str }}=\sqrt{\lambda^{\prime}}+\cdots, \quad a_{\text {str }}=\frac{N}{2 m L}+\cdots, \quad \sqrt{1-\tau}=\frac{L m}{2 N} \sqrt{\lambda^{\prime}}+\cdots,
$$

в то время как выражение для $a_{\mathrm{str}}$ такое же, как и в формуле (3.36). Отсюда можно предположить, что поправки старших порядков при $\xi_{\text {str }} \gg 1$ изменяют только низшую точку разреза. Из (3.35) можно найти, что аномальная размерность имеет масштабное поведение $\gamma(\lambda) \sim m \sqrt{\lambda} \ln (N /(m \sqrt{\lambda}))$ и находится в согласии с выражением (3.21) при $m=1$. Мы можем заключить, что логарифмическое масштабное поведение аномальной размерности при $N \gg L$ с точки зрения струнной теории реализуется, когда нижняя граница $b_{\text {str }}$ разреза приближается к своему минимальному возможному значению $\sqrt{\lambda^{\prime}}$, которое совпадает с положением двойного полюса дифференциала (3.25).

Благодарности. Работа была частично поддержана CRDF (грант CRDF-RUP2261-МО-04) и РФФИ (грант № 04-01-00646).

\section{Список литературы}

[1] J. M. Maldacena, Adv. Theor. Math. Phys., 2 (1998), 231; S.S. Gubser, I. R. Klebanov, A. M. Polyakov, Phys. Lett. B, 428 (1998), 105; E. Witten, Adv. Theor. Math. Phys., 2 (1998), 253.

[2] A.V. Belitsky, A.S. Gorsky, G.P. Korchemsky, Nucl. Phys. B, 748 (2006), 24; hep-th/0601112.

[3] S. Frolov, A. A. Tseytlin, JHEP, 06 (2002), 007; hep-th/0204226. 
[4] S.S. Gubser, I. R. Klebanov, A. M. Polyakov, Nucl. Phys. B, 636 (2002), 99; hep-th/0204051.

[5] D. Berenstein, J. M. Maldacena, H. Nastase, JHEP, 04 (2002), 013; hep-th/0202021.

[6] A. A. Tseytlin, "Spinning strings and AdS/CFT duality", From Fields to Strings: Circumnavigating Theoretical Physics, Ian Kogan memorial collection, vol. 2, eds. M. Shifman, A. Vainshtein, J. Wheater, World Scientific, River Edge, NJ, 2005, 1648; hep-th/0311139.

[7] V.M. Braun, S.E. Derkachov, A. N. Manashov, Phys. Rev. Lett., 81 (1998), 2020; hep-ph/9805225; V. M. Braun, S. E. Derkachov, G. P. Korchemsky, A. N. Manashov, Nucl. Phys. B, 553 (1999), 355; hep-ph/9902375; A. V. Belitsky, Phys. Lett. B, 453 (1999), 59; hep-ph/9902361; Nucl. Phys. B, 558 (1999), 259; hep-ph/9903512; 574 (2000), 407; hep-ph/9907420; S. E. Derkachov, G. P. Korchemsky, A. N. Manashov, Nucl. Phys. B, 566 (2000), 203; hep-ph/9909539.

[8] A. V. Belitsky, V.M. Braun, A. S. Gorsky, G.P. Korchemsky, Int. J. Mod. Phys. A, 19 (2004), 4715; hep-th/0407232.

[9] N. Beisert, M. Staudacher, S. Frolov, A.A. Tseytlin, JHEP, 10 (2003), 037; hep-th/0308117.

[10] R. J. Baxter, Ann. Physics, 70 (1972), 193; Р. Бэкстер, Точно решаемые модели в статистической механике, Мир, М., 1985.

[11] Н. Ю. Решетихин, Ф.А. Смирнов, Записки науч. сем. ЛОМИ, 131 (1983), 128.

[12] G. P. Korchemsky, I. M. Krichever, Nucl. Phys. B, 505 (1997), 387; hep-th/9704079.

[13] M. Kruczenski, JHEP, 08 (2005), 014; hep-th/0410226.

[14] A. V. Belitsky, A.S. Gorsky, G.P. Korchemsky, Nucl. Phys. B, 667 (2003), 3; hep-th/0304028.

[15] И. М. Кричевер, Функи. анализ и его прилож., 28:1 (1994), 26.

[16] V.A. Kazakov, K. Zarembo, JHEP, 10 (2004), 060; hep-th/0410105. 